\title{
Simplified calculation method for noise reduction by barriers on the ground
}

\author{
Lao Kim Leang, ${ }^{*}$ Yasuhiro Yamashita, ${ }^{* *}$ and Masayuki Matsui*** \\ *JMI Institute, \\ 8-12-1, Higashi-ooi, Shinagawa-ku, Tokyo, 140 Japan \\ **Faculty of Engineering, Shinshu University, \\ 500, Wakasato, Nagano, 380 Japan \\ ***Faculty of Engineering, Musashi Institute of Technology, \\ 1-28-1, Tamatsutsumi, Setagaya-ku, Tokyo, 158 Japan
}

(Received 26 June 1989)

\begin{abstract}
This paper introduces a calculation method which evaluates noise reduction caused by barriers considering various ground effects. It is necessary to take the problem of sound diffraction and sound propagation over the ground surface into account in order to obtain the solution of noise reduction by barriers installed on the ground. Our proposing method, in view of practical use, indicates that noise attenuation by diffracted wave and that of ground-reflected wave (including ground wave) are calculable independently, hence the total noise attenuation by barriers on the ground should be presented with a simple numerical expression. Good agreements are found between calculations and model experiments as well as field experiments.
\end{abstract}

Keywords: Noise barrier, Noise attenuation, Acoustic impedance, Flow resistance, Diffraction, Reflection, Interference

PACS number: 43. 20. Fn, 43. 28. Fp

\section{INTRODUCTION}

It is known that the effects of noise barrier installed on the ground are dominated by acoustical properties of the ground. Noise attenuation obtained by the installation of barrier results not only from the effect of diffraction at the edge of the barrier but also from the effect of reflection from the ground on either side of the barrier. Consideration to these phenomena is required to evaluate the barrier effects.

As for the matter of sound diffraction caused by a semi-infinite barrier in the free-field, a number of studies have been done theoretically as well as experimentally, ${ }^{1-3)}$ and also studies have been made on the problem of sound propagation over the ground surface with finite acoustic impedance (without barrier). ${ }^{4-8}$ These theories have been established independently. On the other hand, these theories were simultaneously applied to the mixed problem of the barrier on the ground in some methods such as Jonasson's, ${ }^{9)}$ Thomasson's, ${ }^{10)}$ and Isei's. ${ }^{11)}$

These methods have been established based on the idea of a combination of diffraction theory with the over-ground propagation theory.

The purpose of this paper is to consider the effect of diffracted wave and the effect of ground-reflected wave (including ground wave) separately, and to introduce a simplified calculation method for noise reduction by barriers on the ground for practical use.

\section{SOUND DIFFRACTION BY SEMI-INFINITE BARRIER AND ITS APPLICATION}

Concerning the matter of sound diffraction by a semi-infinite barrier, Maekawa's chart ${ }^{3)}$ and Macdonald's solution ${ }^{2)}$ are used. Maekawa's chart, which was revised through a series of experi- 


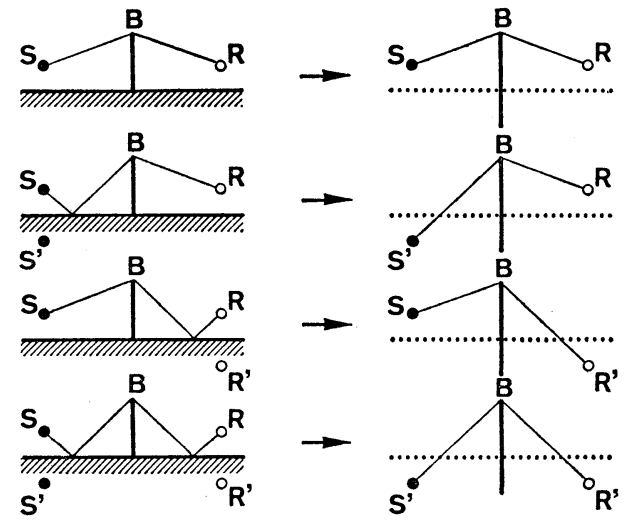

Fig. 1 Sound propagation paths caused by the diffraction by barrier on the ground. $S$ : sound source, $R$ : sound receiver, $B$ : barrier, $S^{\prime}$ : image source, $R^{\prime}$ : image receiver.

ments, has an advantage of the practicability. Macdonald's solution, known as rigorous solution, was selected for a comparison with Maekawa's chart.

Introducing the image source and the image receiver in the ground, we can obtain the sound propagation paths as shown in Fig. 1. Thus, the value of attenuation which corresponds to each path is calculable by using Maekawa's chart or Macdonald's solution. These values are added together in terms of energy to obtain the noise attenuation caused by the diffraction by barrier on the ground: $A T T_{\mathrm{B}}$.

\section{NOISE ATTENUATION OVER THE GROUND (WITH NO BARRIER)}

The sound propagation over the ground surface has been explained by Rudnick ${ }^{4)}$ and Ingard. ${ }^{5)}$ Herein Rudnick's solution is used, and the sound field at the receiver $R$ as shown in Fig. 2 is expressed by Eq. (1) (omitting time dependent term).

$$
\begin{aligned}
\phi & =\phi_{1}+\phi_{2} \\
& =\frac{e^{i k S R}}{S R}+\frac{e^{i k S^{\prime} R}}{S^{\prime} R} \cdot Q
\end{aligned}
$$

Where $k$ is the propagation constant of the air, $Q$ is the image source strength which depends on the ground impedance and the propagation constant of the ground. The image source strength, expressed by $Q=R_{\mathrm{p}}+\left(1-R_{\mathrm{p}}\right) \cdot F(w), R_{\mathrm{p}}$ : plane wave reflection

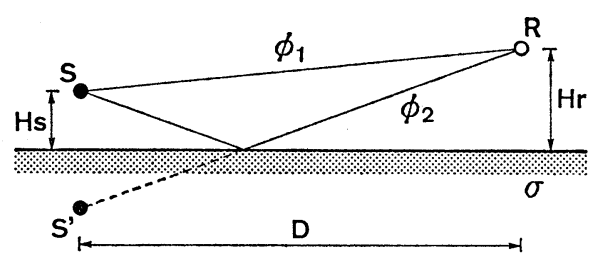

Fig. 2 Sound propagation over the ground surface. $\phi_{1}$ : sound field of direct wave, $\phi_{2}$ : sound field of ground-reffected wave and ground wave. $\sigma$ : flow resistance for the ground.

coefficient, $F(w)$ : boundary loss factor, has been described in detail in Ref. 4).

Considering the physical meaning of the Eq. (1), $\phi_{1}$ is the sound field of direct wave which propagates in the air and $\phi_{2}$ stands for the sound field of the ground-reflected wave and the ground wave.

Accordingly, the attenuation over the ground surface is given by

$$
A T_{\mathrm{G}}=10 \log _{10}\left|\frac{\phi_{1}}{\phi_{1}+\phi_{2}}\right|^{2}
$$

As stated above, it is necessary to obtain the acoustic impedance value of the ground and the propagation constant of the ground as well as geometrical conditions in order to calculate the image source strength $Q$.

Delany and Bazley ${ }^{6)}$ have found experimental relationships between the flow resistance and the propagation constant, and between the flow resistance and the acoustic impedance of porous material. Chessel $^{7)}$ has reported the possibility of the application of these relationships to the propagation constant and the acoustic impedance of the ground. According to Chessell's report and Isei's report, the flow resistance $\sigma$ is estimated at $20,000 \mathrm{cgs}$ units for asphalt and $300 \mathrm{cgs}$ units for grass covered ground.

Therefore, the introduction of the flow resistance is regarded as an efficient method in order to express the acoustical properties of the ground.

Herein, by fitting the calculated results to the experimental results as shown in Fig. 3, we have obtained the values of flow resistance $\sigma$ as follows: $9,500 \mathrm{cgs}$ units for acryl ground and $90 \mathrm{cgs}$ units for flannel ground (values in actual scale, converting from a $1 / 40$ scale model experiments), and $300 \mathrm{cgs}$ units for grass covered ground (by field experiments). ${ }^{12,13)}$ 


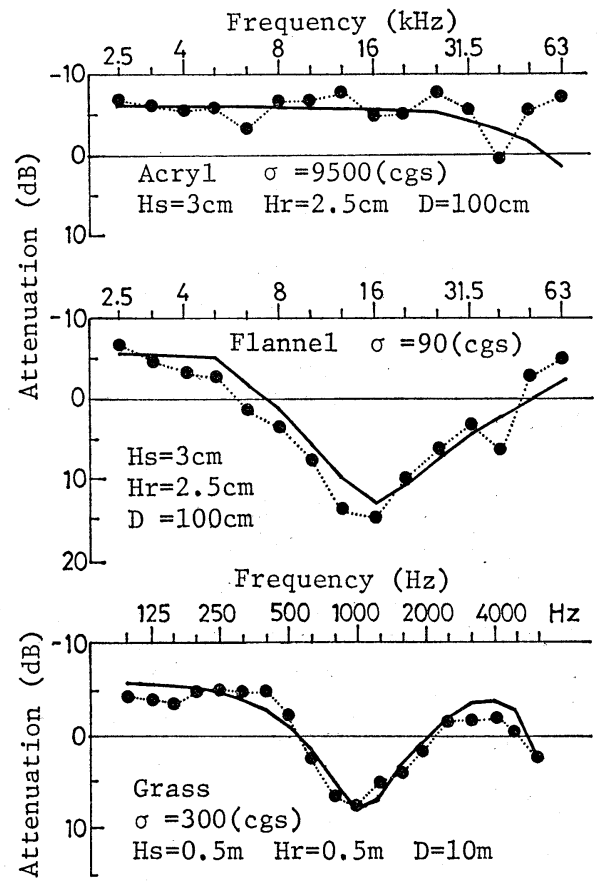

Fig. 3 Flow resistance $\sigma$ of each ground surface by fitting the calculated results to the experimental results. results, _- : calculated curve.

\section{NOISE ATTENUATION OVER THE GROUND WITH THE PRESENCE OF BARRIER}

According to Huygens-Fresnel's principle, the sound diffraction point is regarded as the secondary sound source. As for the sound diffraction by barrier, regarding the edge of barrier as the secondary sound source enables us to separate the sound propagation over the ground on the source side from that on the receiver side of the barrier acoustically, as shown in Fig. 4.

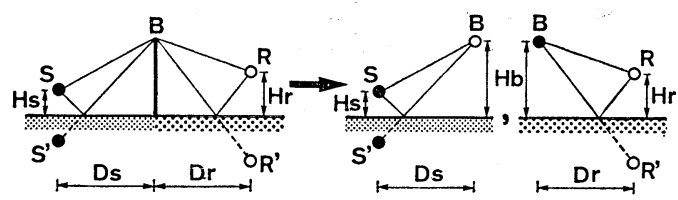

Fig. 4 Geometrical configuration of sound source, barrier and receiver. Simplification of sound propagation over the ground by regarding the edge of barrier as secondary sound source.
Then, the Eq. (1) should be replaced with followings, for the sound source side:

$$
\begin{aligned}
\phi_{\mathrm{S}} & =\phi_{\mathrm{S} 1}+\phi_{\mathrm{S} 2} \\
& =\frac{e^{i k S_{B}}}{S B}+\frac{e^{i k S^{\prime} B}}{S^{\prime} B} \cdot Q_{\mathrm{S}}
\end{aligned}
$$

for the receiver side:

$$
\begin{aligned}
\phi_{\mathrm{R}} & =\phi_{\mathrm{R} 1}+\phi_{\mathrm{R} 2} \\
& =\frac{e^{i k B R}}{B R}+\frac{e^{i k B R^{\prime}}}{B R^{\prime}} \cdot Q_{\mathrm{R}}
\end{aligned}
$$

where $Q_{\mathrm{S}}$ and $Q_{\mathrm{R}}$ are the image source strengths depending on the properties of the ground on the source side and receiver side respectively.

Similarly, as stated in section 3 , the attenuations over the ground on the source side and on the receiver side are given by Eq. (5) and Eq. (6) respectively.

$$
\begin{aligned}
& A T_{\mathrm{GS}}=10 \log _{10}\left|\frac{\phi_{\mathrm{S} 1}}{\phi_{\mathrm{S} 1}+\phi_{\mathrm{S} 2}}\right|^{2} \quad(\mathrm{~dB}) \\
& A T_{\mathrm{GR}}=10 \log _{10}\left|\frac{\phi_{\mathrm{R} 1}}{\phi_{\mathrm{R} 1}+\phi_{\mathrm{R} 2}}\right|^{2} \quad(\mathrm{~dB})
\end{aligned}
$$

Regarding diffraction point as secondary sound source, we have introduced the idea of separating the sound field divided by the barrier. If we consider the fact that these sound fields took place simultaneously and related each other, the attenuation over the ground on both sides should be expressed by using the sum of two coefficients: $\phi_{\mathrm{S} 1}$ $\left(\phi_{\mathrm{S} 1}+\phi_{\mathrm{S} 2}\right)$ and $\phi_{\mathrm{R} 1} /\left(\phi_{\mathrm{R} 1}+\phi_{\mathrm{R} 2}\right)$ as stated in Eqs. (5) and (6), namely

$$
\begin{aligned}
\psi & =\psi_{\mathrm{S}}+\psi_{\mathrm{R}} \\
& =\frac{\phi_{\mathrm{S} 1}}{\phi_{\mathrm{S} 1}+\phi_{\mathrm{S} 2}}+\frac{\phi_{\mathrm{R} 1}}{\phi_{\mathrm{R} 1}+\phi_{\mathrm{R} 2}}
\end{aligned}
$$

Then, the level of noise attenuation $A T T_{\mathrm{G}}$ caused by the presence of ground surface on both sides of the barrier can be given by

$$
A T T_{\mathrm{G}}=10 \log _{10}|\psi|^{2} \quad(\mathrm{~dB})
$$

As for the noise ignoring the phases of $\psi_{\mathrm{s}}$ and $\psi_{R}$, the sum of energy leads following,

$$
|\psi|^{2}=\left|\psi_{\mathrm{S}}\right|^{2}+\left|\psi_{\mathrm{R}}\right|^{2}
$$

Substitution of this value into Eq. (8), $A T T_{\mathrm{G}}$ is obtained by

$$
A T T_{\mathrm{G}}=10 \log _{10}\left[10^{A T_{\mathrm{GS}} / 10}+10^{A T_{\mathrm{GR}} / 10}\right]
$$

Now let us discuss the problem of the total noise attenuation by barrier on the ground. 
We have treated the diffraction phenomenon (at the edge of barrier) and the reflection phenomenon (from the ground on both sides of barrier) independently. Consequently, the attenuation corresponding to each phenomenon has been calculated separately.

Accordingly, the total noise attenuation level $A T T_{\mathrm{B} G}$ can be obtained as the sum of these two attenuation levels, that is

$$
A T T_{\mathrm{BG}}=A T T_{\mathrm{B}}+A T T_{\mathrm{G}} \quad(\mathrm{dB})
$$

where $A T T_{\mathrm{B}}$ is the attenuation by diffraction as stated in section 2, and $A T T_{\mathrm{G}}$ is the attenuation over the ground with the presence of barrier.

\section{EXPERIMENTAL RESULTS AND EVALUATIONS}

A series of model experiments and field experiments were carried out to verify the proposing method. ${ }^{12,13)}$

\subsection{Model Experiments}

Model experiments, whose scale is $1 / 40$, are set in an anechoic room used acryl board barriers on two kinds of model ground surfaces;

1) a $3 \mathrm{~mm}$ thick acryl board is laid over a $12 \mathrm{~mm}$ thick veneer board (hereinafter called acryl ground)

2) a piece of thick flannel wool cloth over the acryl ground (hereinafter called flannel ground)

The flow resistance of these two model ground surfaces are shown as in Fig. 3.

The block diagram of the experimental apparatus is shown as in Fig. 5. A tweeter speaker which

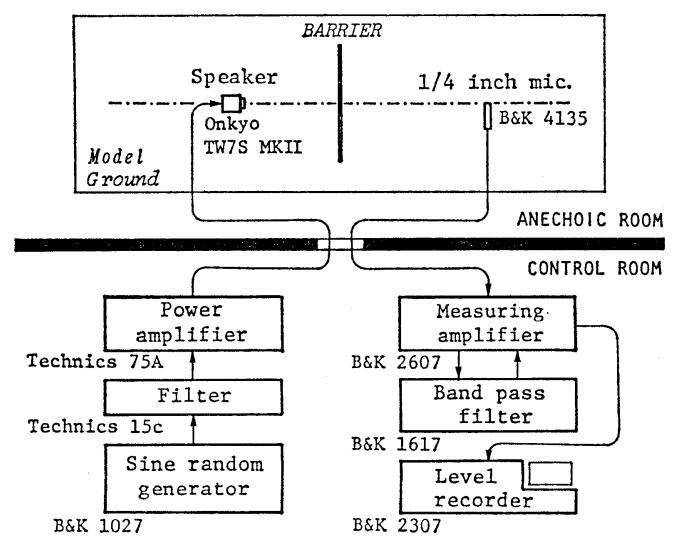

Fig. 5 Block diagram of measurement system. emits pink noise was used for the sound source, and $1 / 4$ inch microphone was installed on the receiver.

Calculations for the noise attenuation caused by
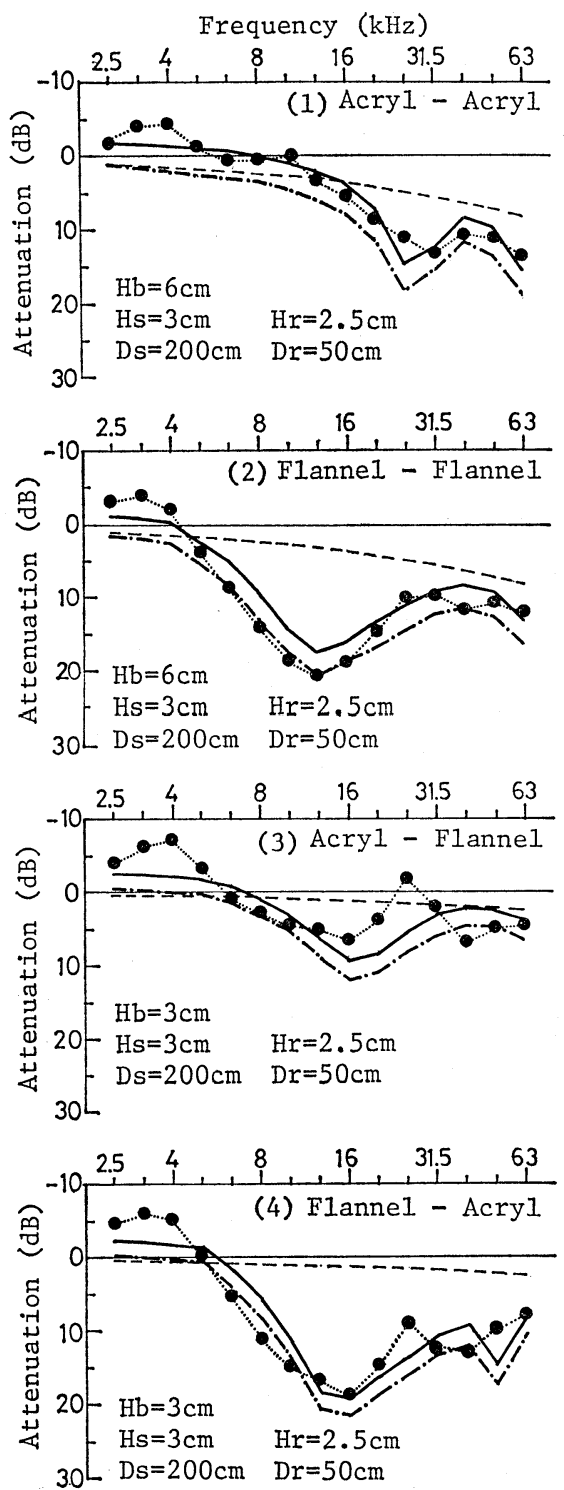

Fig. 6 Comparison between calculated results and model experimental results. experimental results, - - - : attenuation by diffraction contribution only (Maekawa's chart), - : calculated curve by the application of Maekawa's chart and Rudnick's solution, -_-_-_: calculated curve by the application of Macdonald's solution and Rudnick's solution. 


\section{K. LEANG et al.: NOISE REDUCTION BY BARRIERS ON THE GROUND}

the barriers considering ground effect are made based on the Eqs. (10) and (11). Here, we present two applications of the proposing method, first by taking Maekawa's chart for the attenuation by diffraction and Rudnick's solution for the attenuation over the ground on each side of the barrier, secondly by taking Macdonald's solution instead of Maekawa's chart for the attenuation by diffraction.

Figure 6 shows the comparison between calculated results and model experimental results for four combinations of ground surface on source side and receiver side: (1) acryl-acryl, (2) flannel-flannel, (3) acryl-flannel, (4) flannel-acryl.

With the same geometrical configurations, where the ground on both sides are same material: acrylacryl and flannel-flannel, there is a shift of frequency of interference minimum caused by the phase changings on reflection at soft ground surface (flannel ground).

Where the ground material on the source side and on the receiver side are replaced: acryl-flannel and flannel-acryl, there are changings of interference patterns though geometrical configurations are the same.

It is noted that interference minimum pattern shapes due to the presence of flannel ground are wider and deeper than that of the case of acryl ground.

In all these cases, calculated results show good agreements with experimental results though there are some differences in detail. We observe that calculated curves by the application of Macdonald's solution are slightly greater than that obtained by Maekawa's chart. The two calculated curves, however, have almost the same tendency.

\subsection{Field Experiments}

Field experiments are made by using a concrete block barrier on grass covered ground surface whose flow resistance $\sigma$ is $300 \mathrm{cgs}$ units as shown in Fig. 3. A small blower engine, whose muffler was taken off for the improvement of sound power and sound directional characteristics, is used for the sound source.

Here we mention the application case using Maekawa's chart and Rudnick's solution in the proposing method.

Figure 7 shows the comparison between calculated results and field experimental results for different combinations of source and receiver heights. The
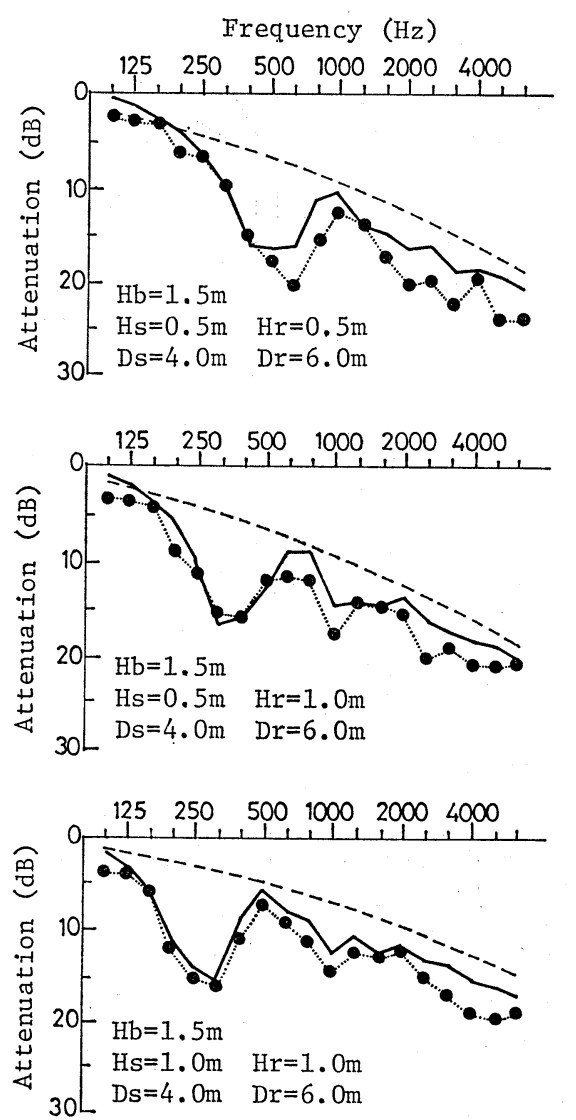

Fig. 7 Comparison between calculated results and field experimental results for different combinations of source and receiver heights. $\bullet$ : experimental results, - - - attenuation by diffraction contribution only (Maekawa's chart), _ $\longrightarrow$ calculated curve by the application of Maekawa's chart and Rudnick's solution.

interference patterns correspond well to the geometrical conditions of each case of experiments. As in model experiments indicated in section 5.1, the results calculated by the proposing method also show good agreement with the experimental results.

We have also made experiments on the effect of oblique incidences represented by $0^{\circ}, 30^{\circ}, 60^{\circ}, 75^{\circ}$ shown as in Fig. 8. Experimental results for each angle of incidence are varying from one to another, and have fluctuation among themselves. Despite the variation of the angle of incidence, these results have similar tendency. Application of Maekawa's chart (disregarding oblique incidence) and Rudnick's 

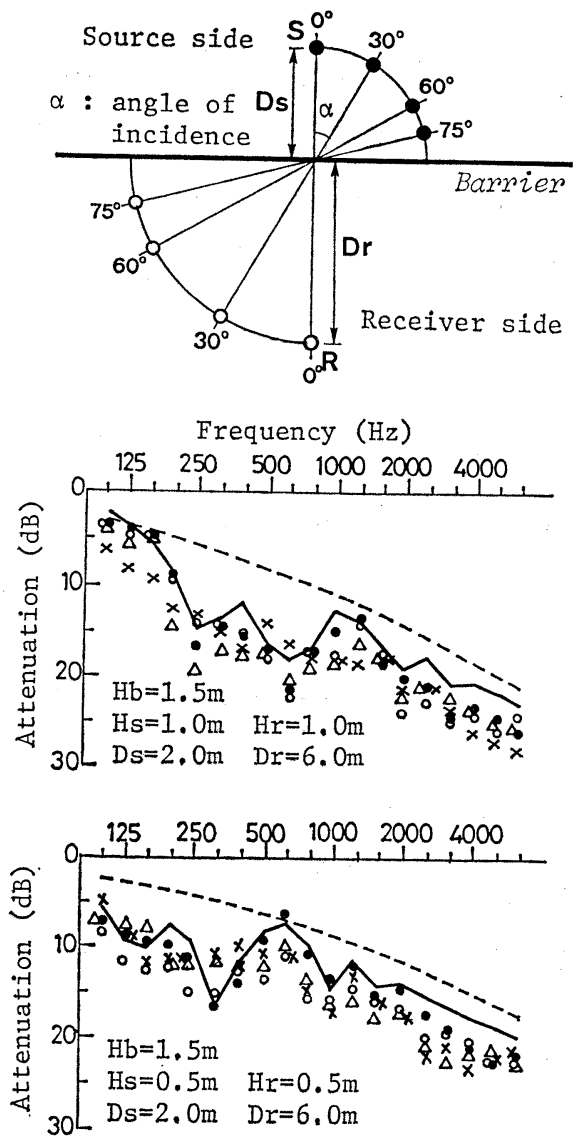

Fig. 8 Comparison between calculated results and field experimental results for different angles of incidence. Experimental results, $\bullet 0^{\circ}, \bigcirc: 30^{\circ}, \triangle: 60^{\circ}, \times 75^{\circ},---$ : attenuation by diffraction contribution only (Maekawa's chart), — : calculated curve by the application of Maekawa's chart and Rudnick's solution.

solution in the proposing method leads us to conclude that our proposing method is practical even if the angle of incidence is large.

\subsection{Comparison of the Proposing Method with an Unsimplified Calculation Method}

We have discussed the problem of noise reduction by barriers on the ground, and have proposed a calculation method for it. Though our proposing method is based on the simplification of numerical expression, it indicates satisfactory agreement with experiments.
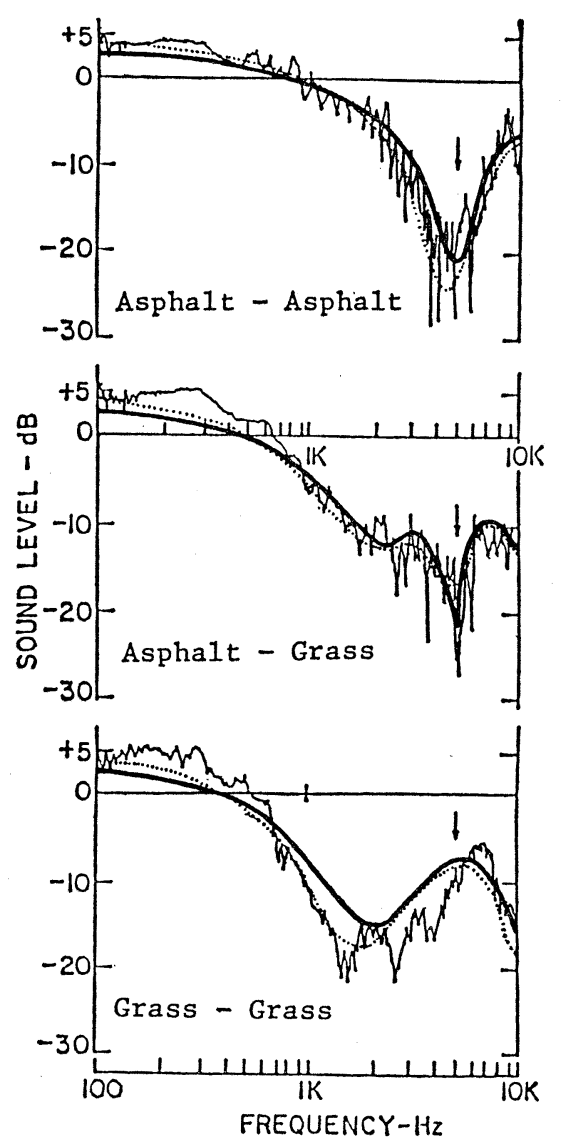

Fig. 9 Comparison of the proposing method with Isei's method and experiments described in Ref. 11). Flow resistance; asphalt: 20,000 (cgs), grass: 300 (cgs), $H b=0.26 \mathrm{~m}, H s=H r=0.125 \mathrm{~m}, D s$ $=D r=2.0 \mathrm{~m}$. - : proposing method, ......: Isei's method, results.

To examine the method in detail, herein, we take one unsimplified calculation method: Isei's method, ${ }^{11)}$ for comparison and verification of our proposing method. Isei's method, based on the combination of sound diffracted wave and sound reflected wave, considers the sound field at receiver as the sum of six components: sound fields due to the real source, its image in the ground, its image in the barrier, and the reflection of these three fields in the ground.

Figure 9 shows the comparison of the two calculation methods with the outdoor experiments carried 


\section{K. LEANG et al.: NOISE REDUCTION BY BARRIERS ON THE GROUND}

out by Isei. ${ }^{11)}$ For the three combinations of ground on source side and receiver side, our proposing method shows close agreement with the unsimplified method, and also with the experimental results. In case of soft ground on each side of the barrier (grassgrass), difference between the two calculations (about $1 \mathrm{kHz}$ to $2 \mathrm{kHz}$ ) is observed, which seems to have been caused by the effect of ground wave, in terms of $\left(e^{i k S^{\prime} B} / S^{\prime} B \cdot Q_{\mathrm{S}}\right) \cdot\left(e^{i k B R^{\prime}} \mid B R^{\prime} \cdot Q_{\mathrm{R}}\right)$, which is ignored in our simplified calculation method.

\section{CONCLUSIONS}

In this report, we have proposed a method for calculating noise reduction by barriers on different ground surfaces. The proposing method is standing on the point that the attenuation by diffraction at the edge of barrier and the attenuation over the ground on both sides of the barrier can be presented separately. Accodringly, the method enables us to solve the complicated problem of barriers on the ground with a simple numerical expression.

We confirmed proposing method could explain the ground-reflected and interference phenomena which related to the properties of the ground, as shown in the comparison of calculated results with model and field experimental results.

This report has presented two applications of the proposing method, one by taking Maekawa's chart for the attenuation by diffraction and Rudnick's solution for the attenuation over ground, another by taking Macdonald's solution instead of Maekawa's chart. Noted that the application of the wellknown Maekawa's chart and Rudnick's solution in the proposing method is good enough to predict the attenuation by barriers on the ground. In principle, other calculations of these attenuation contributions, the attenuation by diffraction and the attenuation over the ground, are also applicable to this method.

From the practical point of view, this calculation method is useful for estimating noise reduction by barriers in the consideration of ground effect, in advantage of its simple numerical expression.

\section{ACKNOWLEDGEMENTS}

We appreciate Dr. Hiroshi Tanaka, Building Research Institute Ministry of Construction, for his kind cooperation providing us an opportunity to use the anechoic room.

\section{REFERENCES}

1) M. Born and E. Wolf, Principles of Optics (Pergamon Press, Oxford, 1980); W. C. Elmore and M. A. Heald, Physics of Waves (McGraw-Hill Kogakusha, Tokyo, 1969).

2) H. M. Macdonald, "A class of diffraction problems," Proc. London Math. Soc. 14, 410-427 (1915).

3) Z. Maekawa, "Noise reduction by screens," Mem. Fac. Eng. Kobe Univ. 11, 29-53 (1965); Appl. Acoust. 1, 157-173 (1968).

4) I. Rudnick, "The propagation of an acoustic wave along a boundary," J. Acoust. Soc. Am. 19, 348-356 (1947).

5) U. Ingard, "On the reflection of a spherical sound wave from an infinite plane," J. Acoust. Soc. Am. 23, 329-335 (1951).

6) M. E. Delany and E. N. Bazley, "Acoustical properties of fibrous absorbent materials," Appl. Acoust. 3, 105-116 (1970).

7) C. I. Chessel, "Propagation of noise along a finite impedance boundary," J. Acoust. Soc. Am. 62, 825834 (1977).

8) Y. Yamashita and M. Matsui, "An excess attenuation of the noise propagation over the ground (Its estimation from field experiments)," J. Acoust. Soc. Jpn. (J) 35, 244-250 (1979) (in Japanese).

9) H. G. Jonasson, "Sound reduction by barriers on the ground," J. Sound Vib. 22, 113-126 (1972).

10) S. I. Thomasson, "Diffraction by a screen above an impedance boundary," J. Acoust. Soc. Am. 63, 1768-1781 (1978).

11) T. Isei, T. F. W. Embleton, and J. E. Piercy, "Noise reduction by barriers on finite impedance ground," J. Acoust. Soc. Am. 67, 46-58 (1980); T. Isei "Absorptive noise barrier on finite impedance ground," J. Acoust. Soc. Jpn. (E) 1, 3-10 (1980).

12) L. K. Leang, Y. Yamashita, N. Takagi, and M. Matsui, "Méthode de calcul de la reduction du bruit due à l'emploi de barrières sur le sol," 11th Int. Congr. Acoust. 8-5-2, 363-366 (Paris) (1983) (in French).

13) L. K. Leang, Y. Yamashita, and M. Matsui, "Effects of diffraction and ground reflection on noise reduction by barriers," Tech. Rep. Noise Acoust. Soc. Jpn. N-8306-3, 15-22 (1983) (in Japanese).

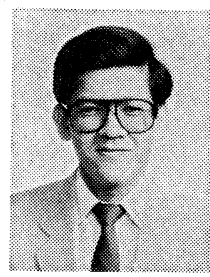

Lao Kim Leang Born Jan. 23rd 1954 in Cambodia. Received Japanese Government (Mombusho) Scholarship from 1974 to 1984 . Graduated from Department of Architecture of Tokyo Institute of Technology (1979). Postgraduated from Department of Environmental Engineering of Tokyo Institute of Technology (1984). Doctor of Engineering. Researcher of JMI Institute. 


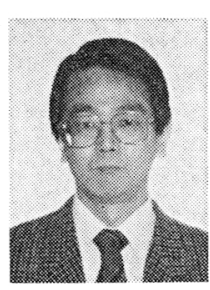

Yasuhiro Yamashita Born Nov. 12th 1942 in Hokkaido. Graduated from Department of Architecture of Nihon University (1968). Doctor of Engineering-Tokyo Institute of Technology (1979). Professor of Shinshu University Department of Architecture \& Civil Engineering.

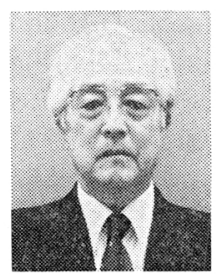

Masayuki Matsui Born Aug. 11th 1923 in Kumamoto Pref. Graduated from Department of Architecture of Tokyo Institute of Technology (1946). Doctor of Engineering-Tokyo Institute of Technology (1961). Professor of Tokyo Institute of Technology (1968-1984). Professor of Shinshu University (1984-1988). Professor of Musashi Institute of Technology (1988- ). Emeritus Professor of Tokyo Institute of Technology (1984). 